\title{
Correlation between the infrared thermogram and microvascular abnormalities of the nailfold in patients with systemic sclerosis
}

\author{
Bartosz Miziołek, Anna Lis-Święty, Alina Skrzypek-Salamon, Ligia Brzezińska-Wcisło \\ Department of Dermatology, School of Medicine, Medical University of Silesia, Katowice, Poland \\ Adv Dermatol Allergol 2020; XXXVIII (1): 115-122 \\ DOI: https://doi.org/10.5114/ada.2021.104286
}

\begin{abstract}
Introduction: Systemic sclerosis (SSc) is a multisystemic disease with an extensive microvasculopathy. The gold standard for its investigation is nailfold videocapillaroscopy (NVC).

Aim: To assess the value of thermography (IRT) for the assessment of microvasculopathy in patients with SSc. Material and methods: Nineteen patients with limited cutaneous SSc were enrolled in the study. They underwent IRT imaging and NVC. An average temperature (Tavg) at the nailfold and a gradient of temperatures $(\Delta$ Tavg) between the central metacarpus of the hand and the nailfold was determined for all fingers. NVC pictures were classified to capillaroscopic patterns according to Cutolo et al. system and they were analysed quantitatively to measure the density of capillaries and to calculate capillaroscopic skin ulcers risk index (CSURI) for each finger separately. Results: There was only a moderate correlation $(0.4<r<0.6)$ between thermographic parameters and density of capillaries in fingers II-V $(r=0.5 ; p<0.001$ for Tavg and $r=-0.45 ; p<0.001$ for $\Delta$ Tavg), but none in thumbs $(r=$ $0.29 ; p=0.089$ for Tavg and $r=-0.19 ; p=0.275$ for $\Delta$ Tavg). Early pattern was associated with a significantly greater surface temperature (Tavg) of nailfolds and essentially milder $\Delta$ Tavg in fingers II-V when compared to all other capillaroscopic patterns in fingers II-V. Surface temperature (Tavg) was significantly lower and $\Delta$ Tavg was markedly more pronounced in fingers II-V with a greater risk of development of digital ulcers (DU) calculated by CSURI. Conclusions: Although IRT measurements correlate only moderately with density of capillaries, this technique seems to be substantial to determine the capillaroscopic pattern and to identify patients at greater risk of DU development.
\end{abstract}

Key words: thermography, capillaroscopy, microvasculopathy, systemic sclerosis.

\section{Introduction}

Systemic sclerosis (SSc) is a multisystemic disease with an extensive microvasculopathy, the first manifestation of which is Raynaud's phenomenon (RP). This is an abnormal response of the microcirculation to cold exposure or emotional events, what causes a sudden ischemia in acral areas of the body [1]. The classic response is triphasic and it is easy to be observed in fingers. A sudden onset of ischemia makes the digits turn white and then blue with their concomitant cooling. While re-warming, there are a rapid reperfusion and a redness of the skin with a frequent feeling of pain in fingers [2]. Structural abnormalities of microvessels impair blood supply to fingers, causing their cooling and blueish colour, also between the subsequent attacks of RP [1]. Insufficient blood supply delays the healing process of the skin what may contribute to the development of digital tip ulcers (DU) in some of SSc patients [3].

Nailfold videocapillaroscopy (NVC) evaluates the shape and distribution of microvessels at the nailfolds and it is the gold standard for the investigation of microvasculopathy in SSc [4]. Microvasculopathy secondary to SSc is known to start from an enlargement of vascular loops with a subsequent development of megacapillaries. Those abnormal vessels disrupt easily, therefore leading to a loss of blood vessels [5]. The next stages in the development of structural abnormalities seen in SSc were classified into capillaroscopic patterns (early, active, late) by Cutolo et al. [6]. A change of capillaroscopic pattern over time allows for a prediction of disease progression $[7,8]$ or an assessment of a response to treatment $[9,10]$.

Address for correspondence: Bartosz Miziołek MD, PhD, Department of Dermatology, School of Medicine, Medical University of Silesia, 20/24 Francuska, 40-067 Katowice, Poland, phone: +48 32-2591-581, e-mail: bmiziolek@gmail.com

Received: 4.07.2019, accepted: 9.09.2019. 
A shift from an early to active or late pattern is associated with the loss of capillaries. A quantitative analysis of vascular loops was postulated to be helpful in a prediction of DU development [11, 12]. Previously, Sebastiani et al. proposed capillaroscopic skin ulcers risk index (CSURI), which predicts DU development within 3 months $[13,14]$.

Infrared thermography (IRT) is an imaging technique which allows for an indirect assessment of blood flow by measuring the surface temperature of the skin [15]. IRT was shown to be a great imaging tool for the diagnosis of RP [16-19], the distinction between primary and secondary RP $[20,21]$ and an evaluation of a response to treatment [22, 23]. Thermographic parameters were also demonstrated to be useful for the prediction of DU development [24] or differentiation between infection and clear ischemic background of DU [25]. Studies which investigate correlations between thermographic parameters and vascular abnormalities seen at the nailfolds are hardly to be found in the literature.

\section{Aim}

The aim of this study was to determine the value of thermographic parameters for an assessment of microvasculopathy in patients with SSc. Specifically, the intention was to determine a correlation between density of capillaries seen in NVC and thermographic measurements as well as their connection to capillaroscopic pattern and CSURI. The secondary aim of the study was to compare IRT and NVC characteristics of thumbs and fingers $\mathrm{I}-\mathrm{V}$.

\section{Material and methods}

\section{Study group}

Patients with SSc from the Department of Dermatology at the School of Medicine in Katowice, Poland, were enrolled to the study between April and September 2018. All of them had to meet classification criteria for SSc established by the European League Against Rheumatism and American College of Rheumatology in 2013 [26]. Only patients with a limited cutaneous subtype of the disease were recruited. To avoid fluctuations of body temperature due to the menstrual cycle, only postmenopausal women were included in the study. Exclusion criteria involved the use of vasodilating drugs (calcium channel blockers, angiotensin receptor blockers, inhibitors of angiotensin-converting enzyme, nitrates, inhibitors of 5-phospodiesterase), cigarette smoking, alcohol abuse, uncontrolled thyroid disease, calcium deposits in the skin of fingers, infection of nailfolds, inflammatory arthropathy of interdigital joints and palmar contracture of fingers not allowing to perform IRT. All participants were asked to follow pre-test instructions, i.e. avoid manicure procedures for at least 2 weeks, alcohol beverages 2 days before, and refrain from sport activities on the day of the examination. Hand washing or application of moisturizing cream as well as caffeine/hot drinks or food intake were not permitted $2 \mathrm{~h}$ prior to the test.

Enrolled subjects gave their written informed consent. The study was previously approved by the local ethical committee at the Silesian School of Medicine in Katowice, Poland, KNW/0022/KB1/115/17.

\section{Infrared thermography}

Firstly, all participants underwent IRT imaging. This was performed in the room specifically designed for an optimal control of temperature, herein fixed at $21^{\circ} \mathrm{C}$ and with humidity of 50-60\%. Patients were earlier acclimatized for at least 15 min prior to testing. IRT imaging was taken with FLIR T420 camera (FLIR Systems AB, Taby, Sweden) that was previously configured to $20-40^{\circ} \mathrm{C}$ temperature range. Cutaneous emissivity (an ability of surface to emit heat at a given temperature) was 0.95. Both hands were first placed on a flat pin pad to take the picture of the dorsal metacarpus and fingers $I I-V$. Then thumbs were put on a round pin plate which allowed for a coverage of fingers II-V laying beneath. All pictures were taken perpendicularly from a dis-
A

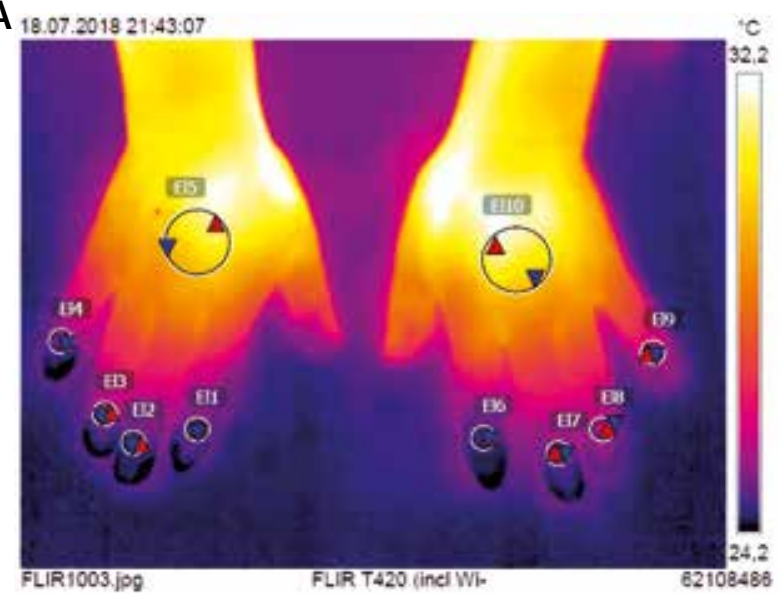

B

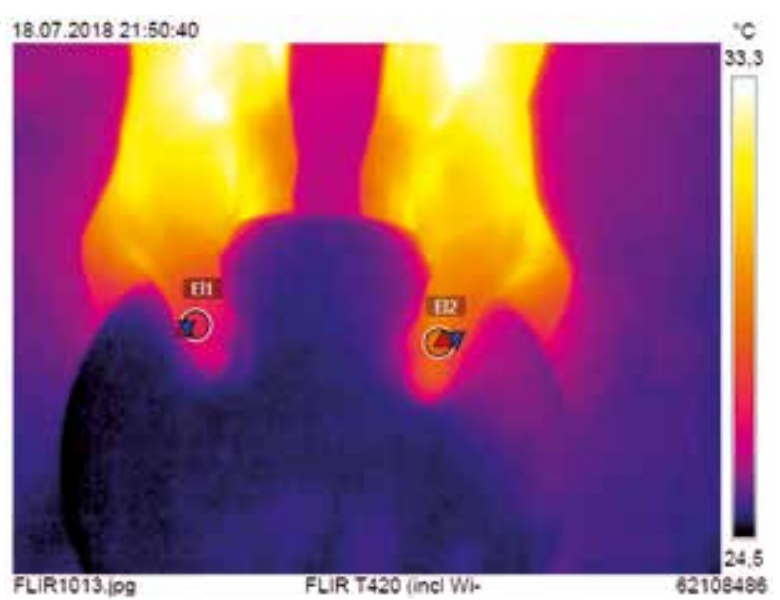

Figure 1. Thermograms with regions of interest set over the nailfolds (ROI1) and over the central metacarpus (ROI2) 
tance of $0.6 \mathrm{~m}$ over hands. They were saved with a resolution of $320 \times 240$ pixels, and then analysed using dedicated software FLIR Tools (v.5.6.16078.1002). Two circular regions of interests (ROI) were determined on the dorsal aspect of the hand. The first one (ROI1) of $10 \mathrm{~mm}$ in diameter was set over the nailfold of each thumb and finger II-V (Figure 1). The second (ROI2) of $30 \mathrm{~mm}$ in diameter was established in the centre of the metacarpus. Minimal (Tmin), maximal (Tmax), and average (Tavg) temperatures were measured for each ROI separately. A gradient of average temperatures ( $\Delta$ Tavg) between the dorsal metacarpus (ROI2) and the nailfold (ROI1) was calculated for each of thumb and finger II-V.

\section{Nailfold videocapillaroscopy}

Following IRT imaging, all nailfolds were investigated with USB digital microscope Dino-Lite MEDL4HMA (AnMo Electronics Corporation, Taiwan). Capillaroscopic pictures were saved with a resolution of $640 \times 480$ pixels. Subsequently, they were analysed using the dedicated software Dino-Capture 2.0 (v.1.5.29.C) after calibration with a scale attached by the device manufacturer. The assessment of microcirculation in nailfolds was performed by two observers (B.M and A.L-Ś). Vascular abnormalities in a patient were classified to capillaroscopic patterns according to Cutolo system [6], and this pattern was next attributed to both thumbs and all fingers $\|-V$ in the same patient. Then, capillaroscopic pictures were submitted to quantitative analysis. The intention was to calculate the density of vascular loops for each of thumbs and fingers II-V as well as to calculate CSURI for each of fingers II-V separately. This mathematical formula (DxM/N2) was calculated basing on a number of megacapillaries (M) multiplied by a diameter of the greatest vascular loop (D) and divided by a square number of capillaries (N2) [13, 14].

\section{Statistical analysis}

Measurements of IRT (Tavg, $\Delta$ Tavg) and NVC (density of capillaries per $1 \mathrm{~mm}$, capillaroscopic pattern, CSURI) were
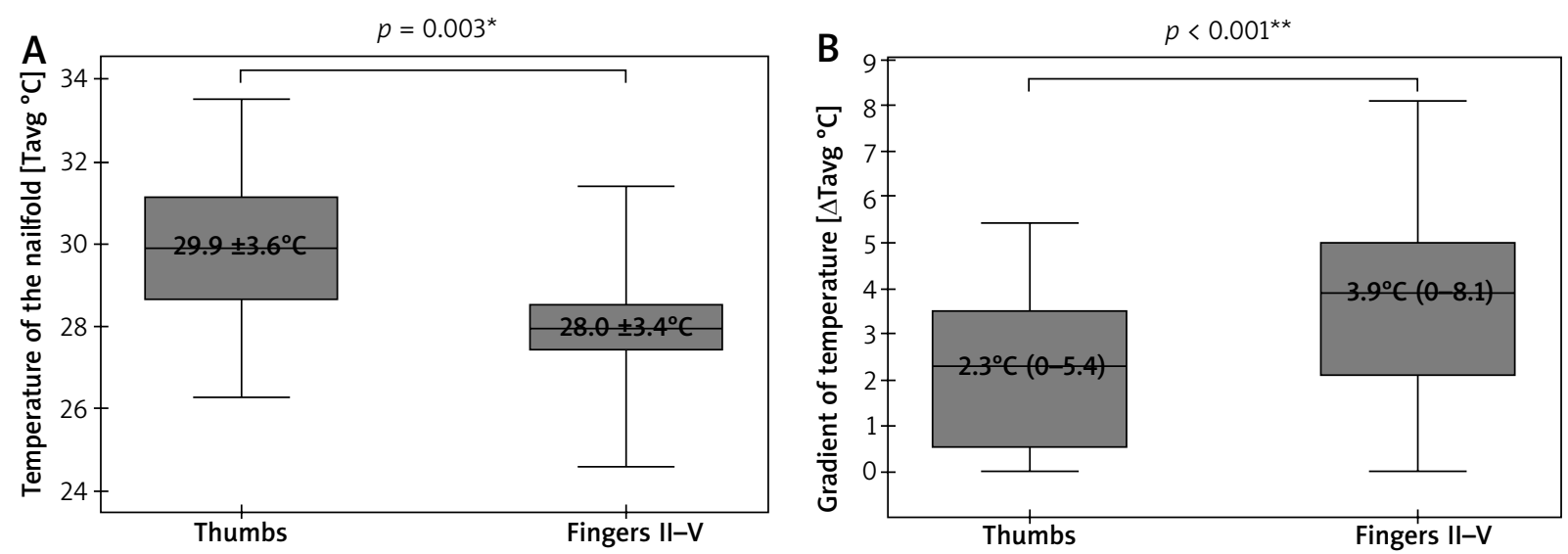

Figure 2. Thermographic differences between thumbs and fingers II-V ( ${ }^{*} t$-Student test, mean $\pm S D ;{ }^{* \star} U$ Mann-Whitney test, median (range)) submitted to statistical analysis with PQStat v.1.6.8.061 (PQStat Software). W Shapiro-Wilk test determined the use of parametric or non-parametric statistics in further analysis of differences between measured values. Non-parametric statistics involved the $U$ Mann-Whitney test to compare two groups, and Kruskal-Wallis test with Dunn-Bonferroni post-hoc analysis in case of three groups. Those parametric statistics included Student's paired t-test and ANOVA test with post-hoc Scheffe test, respectively. A dependence of temperature measurements on the density of capillaries per $1 \mathrm{~mm}$ in the nailfold was checked by Spearman's rank correlation coefficient. A p-value below 0.05 was considered to be statistically significant. Correlation was defined as weak for $r$-value below 0.4, as moderate for $r=0.4-0.6$, and strong for $r$-value greater than 0.6.

\section{Results}

Nineteen patients with SSc were enrolled to the study (4 men and 15 women). Their median age was 58 years (range: $45-65$ years). Anticentromere (ACA) and anti-topoisomerase I (anti-Scl70) autoantibodies were detected in 12 and 7 SSc subjects, respectively. Median duration time of RP was 7 years (range: $1-19$ years) and median duration time of SSc starting from the first nonRP clinical symptom was 5 years (range: $1-12$ years).

A cumulative number of thirty-eight thumbs and one hundred fifty-two fingers II-V underwent IRT and NVC imaging. Due to poor visibility of capillaries in some nailfolds, only thirty-five thumbs and one hundred fortyeight fingers $\|-V$ were included in the final analysis. This revealed significantly lower Tavg in nailfolds of fingers $\|-V$ than in ones of thumbs ( $p=0.003$; $t$-Student), and significantly greater $\Delta$ Tavg in fingers $\|-V$ than in thumbs ( $p<0.001$; U Mann-Whitney) (Figure 2). No temperature gradient between the nailfolds (ROI1) and the central dorsal metacarpus (ROI2) was seen in 8 of 38 thumbs $(21 \%)$ and 8 of 148 fingers II-V (6\%). A density of cap- 
illaries per $1 \mathrm{~mm}$ was significantly greater ( $p=0.006$; $\cup$ Mann-Whitney) in nailfolds of thumbs (median $=7$, range: $3-9)$ than in nailfolds of fingers $\|-V$ (median $=6$, range: $3-9)$.

Spearman's rank correlation coefficient showed only a moderate $(0.4<r<0.6)$ dependence of Tavg measurements at the nailfolds (ROI1) on the density of capillaries per $1 \mathrm{~mm}$ in fingers II-V (Figure $3 \mathrm{~B}$ ). The stronger correlation was found in fingers IV $(r=0.61)$ and $\mathrm{V}$ $(r=0.55)$ and the weaker one was in fingers II $(r=0.44)$ and III ( $r=0.45)$ (Table 1). No significant dependence of Tavg at nailfolds on the density of capillaries was found for thumbs (Figure $3 \mathrm{~A}$ ). Similar analysis for $\Delta$ Tavg showed also its moderate but inverse dependence on the density of capillaries per $1 \mathrm{~mm}$ in fingers $\mathrm{II}-\mathrm{V}$ (Figure $3 \mathrm{D}$ ) with the strongest correlation in fingers IV $(r=-0.54)$ and $V(r=-0.54)$ (Table 1). Once again, there was no dependence of $\Delta$ Tavg at nailfolds on the density of capillaries in thumbs (Figure $3 \mathrm{C}$ ).

NVC pictures were classified as early pattern in $7 \mathrm{pa}-$ tients and this pattern was next attributed to thirteen thumbs and fifty-four fingers $\|-V$ of those patients. Active and late patterns were recognized equally in six subjects, and they were assigned equally to eleven thumbs and forty-seven fingers II-V. IRT imaging demonstrated significantly lower Tavg over the nailfolds in thumbs of patients with late pattern, when compared to ones with early $(p<$ 0.001; ANOVA with post-hoc Scheffe test) or active pattern ( $p=0.045$; ANOVA statistics with post-hoc Scheffe test) (Figure $4 \mathrm{~A}$ ). Statistical analysis of $\Delta$ Tavg in thumbs showed its greater values in thumbs of patients with active as well as late patterns when compared to ones with the early pattern. Herein, a significant difference was found, however, only between the early and late patterns ( $p=0.002$; Kruskal-Wallis with post-hoc Dunn-Bonferroni test) (Figure $4 \mathrm{C}$ ). Similar analysis for fingers II-V demonstrated significant differences of Tavg between all capillaroscopic patterns with the lowest measurement values in fingers of patients with late NVC pattern (Figure 4 B). Differences of $\Delta$ Tavg were also found to be significant between all capillaroscopic patterns for fingers II-V. Herein, the greatest gradient values ( $\Delta$ Tavg) were seen in fingers II-V of patients with late pattern and milder in ones with early or active pattern seen in NVC (Figure 4 D).

The last statistical analysis showed a significant dependence of both Tavg ( $p<0.001$; Student's paired $t$-test) and $\Delta$ Tavg ( $p<0.001$; Student's paired $t$-test) on CSURI (Figure 5). Due to the absence of megacapillaries in NVC of some nailfolds, this risk index was calculated for only 112 of those 148 fingers included in the analysis. Fingers II-V with CSURI $\geq 2.96(n=79)$, suggesting an increased risk of DU development within 3 months, had lower Tavg at the nailfolds as
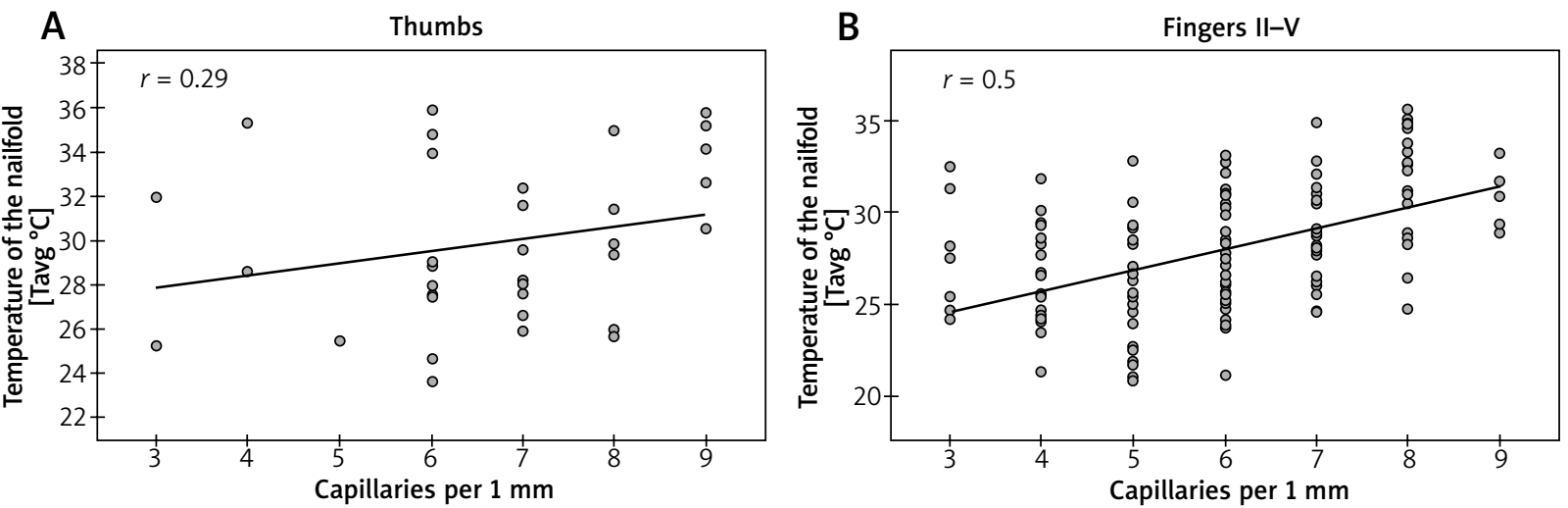

C

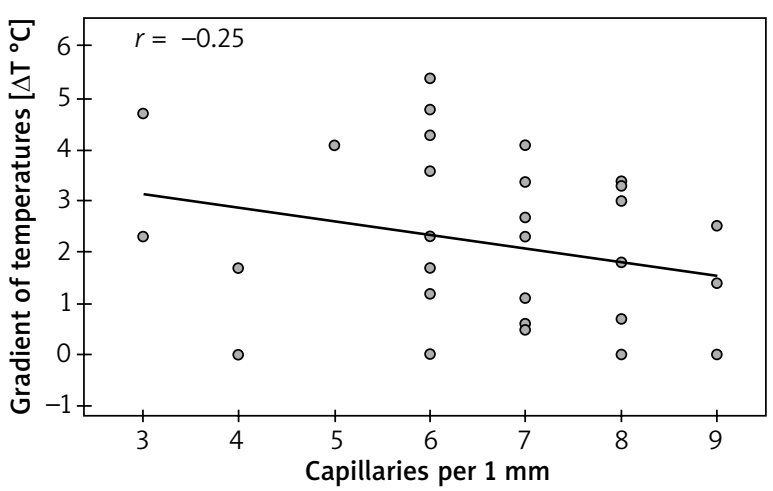

D

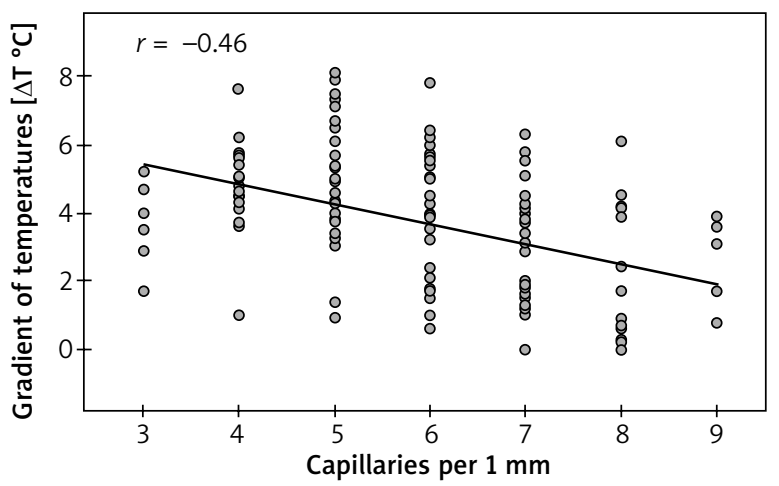

Figure 3. Correlations between thermographic parameters and density of capillaries (Spearman rank correlation coefficient) 
Table 1. Thermographic and capillaroscopic characteristics of fingers II-V

\begin{tabular}{|c|c|c|c|c|c|}
\hline \multirow[t]{2}{*}{ Parameter } & \multicolumn{4}{|c|}{ Fingers II-V } & \multirow[t]{2}{*}{$P$-value } \\
\hline & Index & Middle & Ring & Small & \\
\hline Tavg, mean \pm SD & $28.3 \pm 3.6^{\circ} \mathrm{C}$ & $28.0 \pm 3.4^{\circ} \mathrm{C}$ & $28.0 \pm 3.3^{\circ} \mathrm{C}$ & $28.0 \pm 3.5^{\circ} \mathrm{C}$ & $0.97^{\star}$ \\
\hline$\Delta$ Tavg, median (range) & $3.5 \pm 2.1^{\circ} \mathrm{C}$ & $3.8 \pm 1.9^{\circ} \mathrm{C}$ & $3.7 \pm 1.9^{\circ} \mathrm{C}$ & $3.8 \pm 2.0^{\circ} \mathrm{C}$ & $0.9^{*}$ \\
\hline Capillaries/1 mm, median (range) & 6 & 6 & 5 & 6 & $0.63^{* \star}$ \\
\hline $\begin{array}{l}\text { Capillaries vs. Tavg } \\
\text { rho-Spearman ( } p \text {-value) }\end{array}$ & $0.44(<0.001)$ & $0.45(0.005)$ & $0.61(<0.001)$ & $0.55(<0.001)$ & \\
\hline $\begin{array}{l}\text { Capillaries vs } \Delta \text { Tavg } \\
\text { rho-Spearman ( } p \text {-value) }\end{array}$ & $-0.39(0.02)$ & $-0.4(0.014)$ & $-0.54(<0.001)$ & $-0.54(<0.001)$ & \\
\hline
\end{tabular}

${ }^{*}$ ANOVA statistics, ${ }^{* *}$ Kruskal-Wallis test.
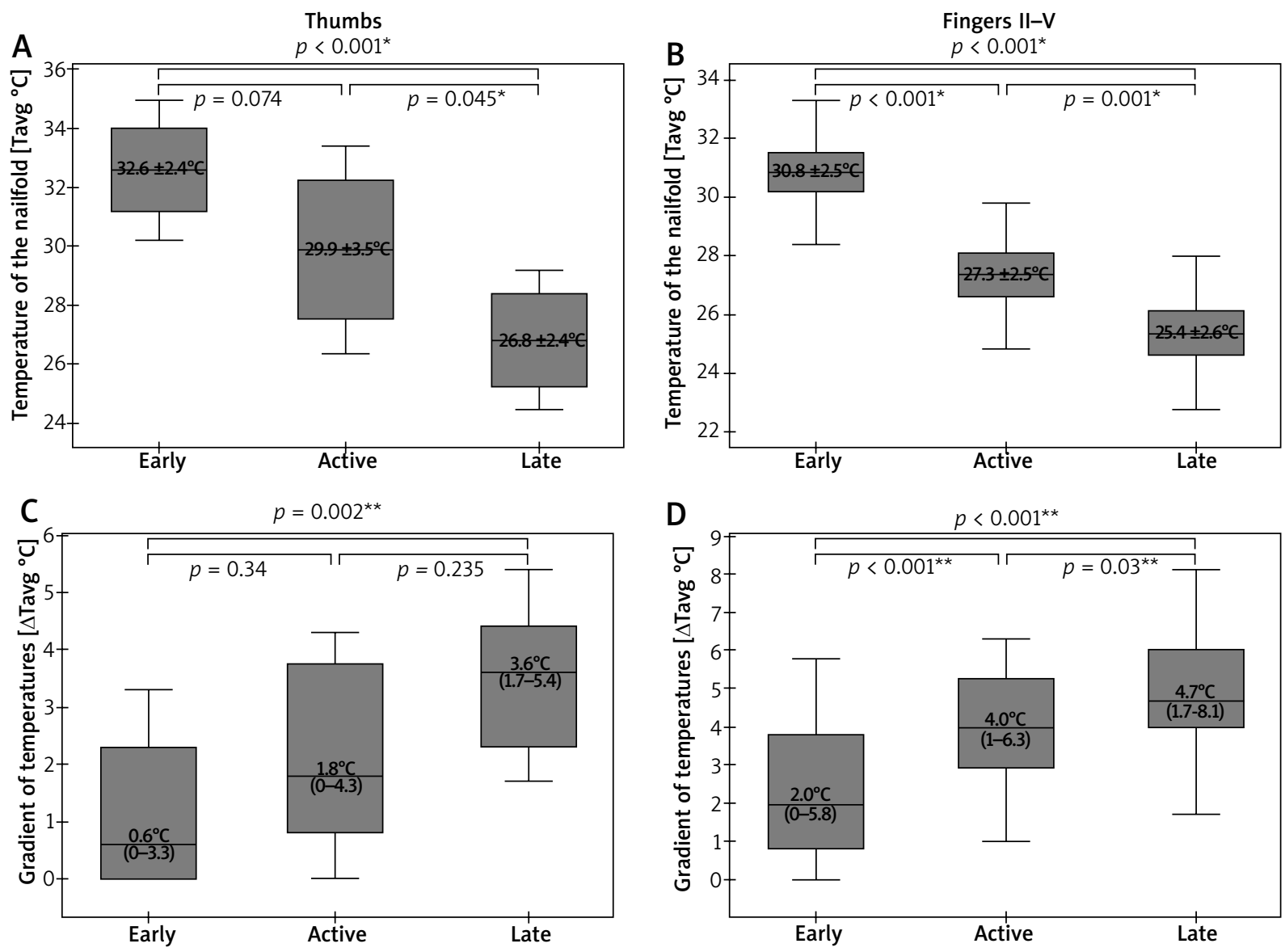

Figure 4. Relationships between thermographic parameters and capillaroscopic pattern ( ${ }^{\star}$ ANOVA statistics with post-hoc Scheffe test, mean \pm SD; ${ }^{* \star}$ Kruskal-Wallis statistics with post-hoc Dunn-Bonferroni test, median (range))

well as significantly more pronounced $\Delta$ Tavg than fingers with CSURI below $2.96(n=33)$.

\section{Discussion}

At present, NVC is a non-invasive diagnostic tool of the greatest significance for the investigation of microvasculopathy. A great value of this method was appreciated by the American College of Rheumatology and the European League Against Rheumatism and thus it was included in classification criteria of SSc in 2013 [26]. The assessment of microcirculation in the nailfold with low-budget USB microscopes or handheld dermoscopes makes NVC more accessible in clinical practice [27]. However, mechanical injuries (e.g. manicure) impair the right interpretation of NVC, whereas skin transparency is often reduced in nonCaucasian subjects or in case of an excessive epidermal scaling and skin thickening $[28,29]$. One must remember that NVC is a subjective diagnostic method for the assessment of microcirculation when there is no quantitative 

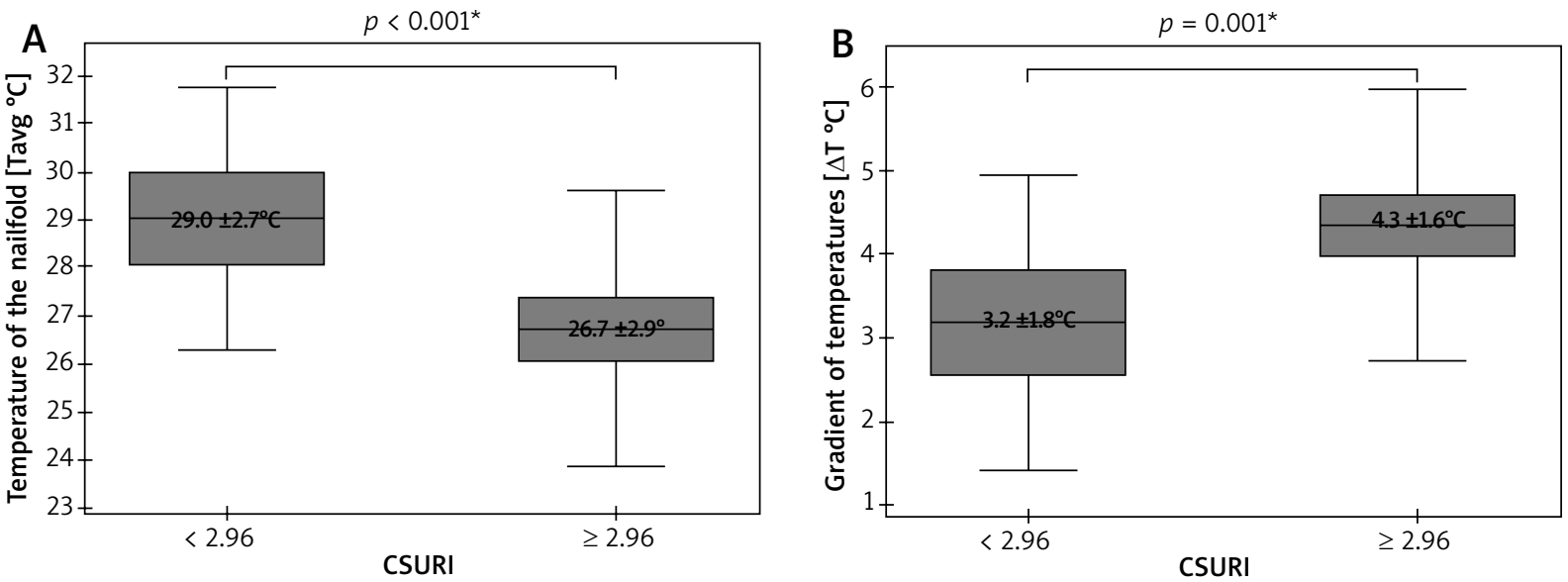

Figure 5. Relationships between thermographic parameters and CSURI ( ${ }^{\star} t$-Student test; mean \pm SD)

analysis and an interpretation of pictures largely depends on the individual observer's experience in NVC [30]. Other non-invasive diagnostic tools helpful in the assessment of microcirculation in SSc patients involve laser Doppler imaging (LDI), laser speckle contrast analysis (LASCA) and IRT. All of them display blood flow in the microcirculation rather than assess structural abnormalities of blood vessels. While LDI and LASCA are poorly available, expensive and time-consuming [31, 32], there is increasing availability of IRT in clinical practice due to falling prices of mobile devices [27].

One of the main aims of our study was to assess an effect of thermographic parameters on the risk of DU development. The risk was evaluated with CSURI that was previously proposed by Sebastiani et al. CSURI was calculated for each of fingers $\|-V$ and they were adapted as separate 'subjects' for imaging. Fingers II-V with a calculated increased risk of new DU development (CSURI $\geq 2.96$ ) were found to be significantly colder than ones without such risk (CSURI < 2.96). This stays indirectly in line with the significantly reduced baseline blood perfusion of the hand what was previously observed by Barbano et al. in SSc patients developing new DU [33]. Reduced acral blood perfusion produces a gradient of temperatures along fingers which herein once again was seen to be greater in subjects (fingers) with CSURI $\geq 2.96$. Persistence of the temperature gradient greater than $1^{\circ} \mathrm{C}$ between one or more fingertips and the dorsum of the hand at $30^{\circ} \mathrm{C}$ after the cold challenge test was earlier reported to be associated with more severe vascular disease, therefore predisposing to trophic lesions on fingertips [24].

A reference of heat emission to capillaroscopic pattern partially confirmed the prediction of decreasing surface temperature at nailfolds and an increasing gradient of temperatures with a progression from early to active and late patterns. Significant differences in both surface temperature and gradient of temperatures were found herein between all capillaroscopic patterns in fingers II-V, but not in case of thumbs. Only thumbs of SSc patients with NVC late pattern showed significantly poor control of surface temperature when compared to ones with early pattern, what produced a significantly greater gradient of temperatures along those thumbs. There was also a significant difference in surface temperature at nailfolds between thumbs with active and late patterns, but it did not impact the occurrence of the significant gradient of temperatures along thumbs. Heat control of thumbs appears therefore to be resistant to the loss of vascular loops with the progression from early to active pattern and its significant dysregulation occurs only when there is severe avascularisation like in case of late pattern.

Furthermore, our study showed moderate dependence of the surface temperature (Tavg and $\Delta$ Tavg) of hands on the density of capillaries measured by NVC technique. Stronger dependence was found in fingers IV-V and weaker in fingers II-III, with almost complete lack of such dependence in thumbs. A distinct behaviour of thumbs in control of temperature was previously reported by Chikura et al. [34, 35]. There are studies which omit measurements of temperature at thumbs $[16,18$, $20,24]$ or analyse perfusion of only fingers II-IV [17, 33]. Herein, measurements of both fingers $I-V$ and thumbs were included, which remains similar to imaging protocols of Merla et al. [21] and Mariotti et al. [36], whose studies also investigated temperature at all five fingers.

Circulation in thumbs appears to be the least dependent on the density of capillaries among all fingers, probably due to anatomical differences of blood supply by arterial structures [37]. Nailfolds of thumbs were found herein to be significantly warmer than ones of fingers II-V what was associated with an essentially milder gradient along thumbs than along other fingers. Thumbs 
were also found to be less susceptible to the loss of microvascular loops and their density of capillaries herein was significantly greater than in fingers $\|-V$. Interestingly, the index finger was seen to be slightly warmer than other three fingers III-V (although insignificantly). The index finger partially shares blood supply with thumbs through arteria princeps pollicis, which probably transmits the pressure of blood stream directly from the radial artery [38]. Previously, index fingers together with thumbs were found by Merla et al. to have almost normal thermoregulatory response in SSc patients whereas this was abnormal in fingers III-V [21].

A stronger correlation between temperature and capillary density was seen in fingers IV-V, and weaker in fingers II-III. A potential explanation is an occlusion of the ulnar artery which develops in some of SSc patients [39-41]. This would make blood circulation in fingers IV-V more dependent on the palmar arch supplied by the radial artery. A weaker blood flow from the ulnar area of the hand may potentially produce a greater dependence of blood supply in fingers IV-V on the density of capillaries, in contrast to thumbs and fingers II-III, which are located closer to the radial artery. The strongest dependence was found for finger IV and, therefore, its thermal control is more susceptible to a loss of capillaries in contrast to other fingers of the hand. Previously, the fourth finger was even identified to be the coolest one among fingers II-IV in both healthy controls and patients with RP [17].

Structural abnormalities of the microcirculation cause large diversity in regional thermoregulatory behaviour across fingers of the same hand. This may produce irregular spots of different heat emission [21] or a thermal asymmetry between both hands and fingers [16]. Recovery of blood flow to acral regions is frequently incomplete following the RP attack in SSc settings, what produces a persistently increased gradient of temperatures along fingers $[20,24]$. Heterogeneity in response to cold or heat exposure enables considering measurements of temperature separately for each finger instead of treating them collectively. Distinct behaviour of microcirculation across fingers was used in the study to make each thumb and finger a separate 'subject' for imaging. Capillaroscopic pattern in a given patient was attributed to all his/her thumbs and fingers. This enabled an analysis of temperature dependence on capillaroscopic pattern in a large group of thumbs and fingers instead of only a small study cohort.

A critical point for the study was the choice of ROI for IRT measurements, which was established on the dorsal aspect of hands over the nailfolds and the central metacarpus. Previous authors set ROI within the nail-bed region of all five fingers [36], palmar surface of fingertips [18], fingertips and the central metacarpus [17], whole dorsal surface of the metacarpus and fingers II-V [16], volar surface of hands [23] or even both aspects of the hand [15]. Calculation of the difference between the central meta- carpus and distal areas over nailfolds herein is consistent with measurements of distal-to-dorsal difference proposed earlier by several other authors for RP settings [16, 17, 42]. A gradient of temperatures between two points or areas is, indeed, less influenced by room temperature than measurements involving only a single point or area [19]. Undoubtedly, a great limitation of this study was diverse duration time of RP and SSc. Previously, a negative correlation $(r=-0.47 ; p<0.05)$ between temperature measurements of the digits and the duration time of SSc was indeed reported by Chlebicka et al. [16].

\section{Conclusions}

IRT may be helpful in the evaluation of microvasculopathy in SSc. Although there is only a moderate correlation between the density of capillaries and thermographic parameters, IRT may facilitate observation of the progression from early to active and late patterns typically seen in NVC. This imaging technique seems also to be another promising tool in the prediction of DU development. Finally, thumbs manifest distinct behaviour in SSc. They are less susceptible to the loss of capillaries and their thermoregulatory control is better than in other fingers of the hand, what suggests sparing their analysis with IRT as seen in NVC, for clinical purposes.

\section{Acknowledgments}

This study was funded by statutory grant KNW-1099/N/8/K awarded by the Medical University of Silesia in Katowice.

\section{Conflict of interest}

The authors declare no conflict of interest.

\section{References}

1. Herrick AL The pathogenesis, diagnosis and treatment of Raynaud phenomenon. Nat Rev Rheumatol 2012; 8: 469-79.

2. Cooke JP, Marshall JM. Mechanisms of Raynaud's disease. Vasc Med 2005; 10: 293-307.

3. Nihtyanova SI, Brough GM, Black CM, Denton CP. Clinical burden of digital vasculopathy in limited and diffuse cutaneous systemic sclerosis. Ann Rheum Dis 2008; 67: 120-3.

4. Pizzorni C, Sulli A, Smith V, et al. Capillaroscopy 2016: new perspectives in systemic sclerosis. Acta Reumatol Port 2016; 41: 8-14.

5. Ruaro B, Sulli A, Smith V, et al. Advances in nailfold capillaroscopic analysis in systemic sclerosis. J Scleroderma Relat Disord 2018; 3: 122-31.

6. Cutolo M, Sulli A, Pizzorni C, Accardo S. Nailfold videocapillaroscopy assessment of microvascular damage in systemic sclerosis. J Rheumatol 2000; 27: 155-60.

7. Ingegnoli F, Ardoino I, Boracchi P, Cutolo M. Nailfold capillaroscopy in systemic sclerosis: data from the EULAR scleroderma trials and research (EUSTAR) database. Microvasc Res 2013; 89: 122-8. 
8. Smith V, Riccieri V, Pizzorni C, et al. Nailfold capillaroscopy for prediction of novel future severe organ involvement in systemic sclerosis. J Rheumatol 2013; 40: 2023-8.

9. Aschwanden M, Daikeler T, Jaeger KA, et al. Rapid improvement of nailfold capillaroscopy after intense immunosuppression for systemic sclerosis and mixed connective tissue disease. Ann Rheum Dis 2008; 67: 1057-9.

10. Caramaschi P, Volpe A, Pieropan S, et al. Cyclophosphamide treatment improves microvessel damage in systemic sclerosis. Clin Rheumatol 2009; 28: 391-5.

11. Smith V, De Keyser F, Pizzorni C, et al. Nailfold capillaroscopy for day-to-day clinical use: construction of a simple scoring modality as a clinical prognostic index for digital trophic lesions. Ann Rheum Dis 2011; 70: 180-3.

12. Cutolo M, Herrick AL, Distler O, et al. Nailfold videocapillaroscopic features and other clinical risk factors for digital ulcers in systemic sclerosis: a multicenter, prospective cohort study. Arthritis Rheumatol 2016; 68: 2527-39.

13. Sebastiani M, Manfredi A, Colaci M, et al. Capillaroscopic skin ulcer risk index: a new prognostic tool for digital skin ulcer development in systemic sclerosis patients. Arthritis Rheum 2009; 61: 688-94.

14. Sebastiani M, Manfredi A, Vukatana G, et al. Predictive role of capillaroscopic skin ulcer risk index in systemic sclerosis: a multicentre validation study. Ann Rheum Dis 2012; 71: 67-70.

15. Hwang Y, Seo M, Seo JM. Development of hand blood circulation measurement system for Raynaud syndrome using infrared imaging. Conf Proc IEEE Eng Med Biol Soc 2017; 2017: 1352-5.

16. Chlebicka I, Matusiak L, Maj J, et al. Freezing fingers syndrome, primary and secondary Raynaud's phenomenon: characteristic features with hand thermography. Acta Derm Venereol 2013; 93: 428-32.

17. Lim MJ, Kwon SR, Jung KH, et al. Digital thermography of the fingers and toes in Raynaud's phenomenon. J Korean Med Sci 2014; 29: 502-6.

18. Cherkas LF, Carter L, Spector TD, et al. Use of thermographic criteria to identify Raynaud's phenomenon in a population setting. J Rheumatol 2003; 30: 720-2.

19. Watanabe I, Sagawa A, Baba Y, et al. Thermography of collagen diseases with Raynaud's phenomenon. Ryumachi 1991; 31: 167-74.

20. Anderson ME, Moore TL, Lunt M, Herrick AL. The 'distaldorsal difference': a thermographic parameter by which to differentiate between primary and secondary Raynaud's phenomenon. Rheumatology 2006; 46: 533-8.

21. Merla A, Di Donato L, Di Luzio S, et al. Infrared functional imaging applied to Raynaud's phenomenon. IEEE Eng Med Biol Mag 2002; 21: 73-9.

22. al-Awami M, Schillinger M, Maca T, et al. Low level laser therapy for treatment of primary and secondary Raynaud's phenomenon. Vasa 2004; 33: 25-9.

23. Selenko-Gebauer N, Duschek N, Minimair G, et al. Successful treatment of patients with severe secondary Raynaud's phenomenon with the endothelin receptor antagonist bosentan. Rheumatology 2006; 45 (Suppl 3): 45-8.

24. Hughes M, Wilkinson J, Moore T, et al. Thermographic abnormalities are associated with future digital ulcers and death in patients with systemic sclerosis. J Rheumatol 2016; 43: 1519-22.

25. van der Weijden MAC, van Vugt LM, Valk D, et al. Exploring thermography: a promising tool in differentiation between infection and ischemia of the acra in systemic sclerosis. Int J Rheum Dis 2017; 20: 2190-3.

26. van den Hoogen F, Khanna D, Fransen J, et al. 2013 classification criteria for systemic sclerosis: an American college of rheumatology/European league against rheumatism collaborative initiative. Ann Rheum Dis 2013; 72: 1747-55.

27. Herrick AL, Murray A. The role of capillaroscopy and thermography in the assessment and management of Raynaud's phenomenon. Autoimmun Rev 2018; 17: 465-72.

28. Chojnowski MM, Felis-Giemza A, Olesinska M. Capillaroscopy - a role in modern rheumatology. Reumatologia 2016; 54: 67-72.

29. Cutolo M, Sulli A, Smith V. How to perform and interpret capillaroscopy. Best Pract Res Clin Rheumatol 2013; 27: 237-48.

30. Rodriguez-Reyna TS, Bertolazzi C, Vargas-Guerrero A, et al. Can nailfold videocapillaroscopy images be interpreted reliably by different observers? Results of an inter-reader and intra-reader exercise among rheumatologists with different experience in this field. Clin Rheumatol 2019; 38: 205-10.

31. Eriksson S, Nilsson J, Sturesson C. Non-invasive imaging of microcirculation: a technology review. Med Devices 2014; 7: 445-52.

32. Allen J, Howell K. Microvascular imaging: techniques and opportunities for clinical physiological measurements. Physiol Meas 2014; 35: 91-141.

33. Barbano B, Marra AM, Quarta S, et al. In systemic sclerosis skin perfusion of hands is reduced and may predict the occurrence of new digital ulcers. Microvasc Res 2017; 110: 1-4.

34. Chikura B, Moore TL, Manning JB, et al. Sparing of the thumb in Raynaud's phenomenon. Rheumatology 2008; 47: 219-21.

35. Chikura B, Moore T, Manning JB, et al. Thumb involvement in Raynaud's phenomenon as an indicator of underlying connective tissue disease. J Rheumatol 2010; 37: 783-6.

36. Mariotti A, Grossi G, Amerio P, et al. Finger thermoregulatory model assessing functional impairment in Raynaud's phenomenon. Ann Biomed Eng 2009; 37: 2631-9.

37. Chikura B, Moore T, Manning JB, et al. Comment on: Sparing of the thumb in Raynaud's phenomenon: reply. Rheumatology 2008; 47: 1260 .

38. Miletin J, Sukop A, Baca V, Kachlik D. Arterial supply of the thumb: systemic review. Clin Anat 2017; 30: 963-73.

39. Taylor MH, McFadden JA, Bolster MB, Silver RM. Ulnar artery involvement in systemic sclerosis (scleroderma). J Rheumatol 2002; 29: 102-6.

40. Frerix M, Stegbauer J, Dragun D, et al. Ulnar artery occlusion is predictive of digital ulcers in SSc: a duplex sonography study. Rheumatology 2012; 51: 735-42.

41. Lescoat A, Yelnik CM, Coiffier G, et al. Ulnar artery occlusion and severity markers of vasculopathy in systemic sclerosis: a multicenter cross-sectional study. Arthritis Rheumatol 2019; 71: 983-90

42. Herrick AL, Clark S. Quantifying digital vascular disease in patients with primary Raynaud's phenomenon and systemic sclerosis. Ann Rheum Dis 1998; 57: 70-8. 